\title{
Quantitative proteomics analysis of Mycoplasma pneumoniae identifies potential macrolide resistance determinants
}

\author{
Shaoli Li ${ }^{1}$, Guanhua Xue ${ }^{1 *}$, Hanqing Zhao ${ }^{1}$, Yanling Feng ${ }^{1}$, Chao Yan ${ }^{1}$, Jinghua Cui ${ }^{1}$, Xianghui Xie ${ }^{2 *}$ \\ and Jing Yuan ${ }^{1 *}$
}

\begin{abstract}
Mycoplasma pneumoniae is one of the leading causes of community-acquired pneumonia in children and adolescents. Because of the wide application of macrolides in clinical treatment, macrolide-resistant M. pneumoniae strains have become increasingly common worldwide. However, the molecular mechanisms underlying drug resistance in M. pneumoniae are poorly understood. In the present work, we analyzed the whole proteomes of macrolidesensitive and macrolide-resistant strains of M. pneumoniae using a tandem mass tag-labeling quantitative proteomic technique, Data are available via ProteomeXchange with identifier PXD022220. In total, 165 differentially expressed proteins were identified, of which 80 were upregulated and 85 were downregulated in the drug-resistant strain compared with the sensitive strain. Functional analysis revealed that these proteins were predominantly involved in protein and peptide biosynthesis processes, the ribosome, and transmembrane transporter activity, which implicates them in the mechanism(s) of resistance of $M$. pneumoniae to macrolides. Our results provide new insights into drug resistance in M. pneumoniae and identify potential targets for further studies on resistance mechanisms in this bacterium.
\end{abstract}

Keywords: Mycoplasma pneumoniae, Drug resistance, Whole proteomes, Quantitative proteomic technique, Differentially expressed proteins

\section{Key Points}

1. Macrolide-resistant M. pneumoniae infections are very common worldwide.

2. Quantitative proteomic analysis of macrolide resistance of in M. pneumoniae.
*Correspondence: xgh618@163.com; xiexianghui@bch.com.cn; yuanjing6216@163.com

${ }^{1}$ Department of Bacteriology, Capital Institute of Pediatrics, No. 2 Yabao Road, Chaoyang District, Beijing 100020, China

${ }^{2}$ Department of Urinary Surgery, Capital Institute of Pediatrics, No. 2 Yabao Road, Chaoyang District, Beijing 100020, China

\section{Introduction}

Mycoplasma pneumoniae causes community-acquired pneumonia in children and adolescents (Saraya 2016). Outbreaks of $M$. pneumoniae infections occur every 3-7 years, and $50-80 \%$ of individuals in schools and other semi-enclosed spaces are affected by them. With its ability to survive independently in vitro and with no cell wall, M. pneumoniae, a small prokaryotic bacterium, is naturally resistant to drugs that act on cell walls (Waites et al. 2017). Antibiotics that affect the synthesis of bacterial DNA and protein, such as macrolides, quinolones, and tetracycline, can be used to treat $M$. pneumoniae infections. However, tetracycline can cause tooth yellowing, enamel underdevelopment, gastrointestinal tract stimulant reactions, liver toxicity and other side effects, contraindicating its use for children under 8 years of age. 
Quinolones also cannot be used in children because they can damage cartilage and joints. Therefore, macrolides are currently the first choice treatments for M. pneumoniae infections in children (Lee et al. 2018).

Unfortunately, the widespread clinical application of macrolides has triggered microbial resistance to these agents from the 1970s onwards and, since 2000, macrolide-resistant $M$. pneumoniae strains have become increasingly common in many countries, with drug resistance rates reaching $100 \%$ in some areas, thereby posing a significant threat to human health (Tanaka et al. 2017; Cao et al. 2017). Previous studies on drug resistance in M. pneumoniae have focused on point mutations in the $23 \mathrm{~S}$ ribosomal gene and L4 and L22 ribosomal proteins, but whether or not changes at the protein level contribute to macrolide resistance awaits investigation (Pereyre et al. 2016; Yang et al. 2017). Here, we used a tandem mass tag (TMT)-labeling-based quantitative proteomic technique to identify differentially expressed proteins (DEPs) in macrolide-sensitive versus macrolideresistant M. pneumoniae.

\section{Materials and methods \\ Chemicals}

RIPA Lysis and Extraction Buffer, a TMT10plex Isobaric Label Reagent Set, and a Pierce ${ }^{\mathrm{TM}}$ BCA Protein Assay Kit were purchased from Thermo Fisher Scientific. Urea, triethylammonium bicarbonate (TEAB) buffer $(1.0 \mathrm{M}$, $\mathrm{pH} 8.5 \pm 0.1$ ), Tris (2-carboxyethyl) phosphine (TCEP) hydrochloride solution $(0.5 \mathrm{M}, \mathrm{pH} 7.0)$, iodoacetamide (IAA), formic acid (FA), acetonitrile (ACN), and methanol were purchased from Sigma (St. Louis, MO, USA). Trypsin from bovine pancreas was purchased from Promega (Madison, WI, USA). Ultrapure water was prepared using a Millipore purification system (Billerica, MA, USA).

\section{Strains}

Macrolide-resistant M. pneumoniae strain C267 (GenBank No. CP014267), which was isolated in Beijing, China (Li et al. 2016) and the macrolide-sensitive M129 reference strain (ATCC29342) were used in this study. Two strains were cultured in PPLO broth (Becton, Dickinson and company, USA), yeast extract (10\%, Oxoid LTD, England), unheated horse serum (20\%, Lanzhou national Hyclone Bio-Engineering Co.LTD, China), glucose (50\%, CR Double-Crane Pharmaceuticals Co., Ltd, China), phenol red (0.4\%, Amresco, OH, USA), and penicillin (1000 U/mL, North China pharmaceutical Group Corporation, China) at $37{ }^{\circ} \mathrm{C}$ in a BSL-2 laboratory several days until the color changes. Harvest for protein extraction when the strains $(50 \mathrm{~mL})$ had reached logarithmic growth (color changes occurred within 2-3 days after passage).

\section{Protein extraction and digestion}

Proteins were extracted using RIPA Lysis and Extraction Buffer. Protein concentrations were measured using the Pierce BCA Protein Assay Kit. Protein $(100 \mu \mathrm{g})$ was diluted with $100 \mathrm{mM}$ TEAB to a final volume of $100 \mu \mathrm{L}$. TCEP $(10 \mathrm{mM})$ was added to each sample tube and the mixtures were reacted at $56{ }^{\circ} \mathrm{C}$ for $1 \mathrm{~h}$. Proteins were alkylated using $20 \mathrm{mM}$ IAA at room temperature in the dark for $1 \mathrm{~h}$. Pre-chilled acetone $\left(-20{ }^{\circ} \mathrm{C}, 180 \mu \mathrm{L}\right)$ was added and the mixture was stored at $-20^{\circ} \mathrm{C}$ overnight. Samples were centrifuged at $8000 \times g$ for $10 \mathrm{~min}$ at $4{ }^{\circ} \mathrm{C}$. The acetone was carefully removed without disturbing the white pellet and the pellet was allowed to dry for 2-3 $\mathrm{min}$. The acetone-precipitated protein pellet $(100 \mu \mathrm{g})$ was resuspended in $100 \mu \mathrm{L}$ of $50 \mathrm{mM}$ TEAB. Free trypsin $(2 \mu \mathrm{g})$ was added to the protein solution and the solution was incubated at $37{ }^{\circ} \mathrm{C}$ overnight. Each experiment was repeated three times.

\section{Labeling and peptide fractionation}

Immediately before use, the TMT labeling reagents were equilibrated to room temperature. Anhydrous ACN (41 $\mu \mathrm{L}$ ) was added to each tube and the reagent was allowed to dissolve for $5 \mathrm{~min}$ with occasional vortexing. The samples were labeled with the TMT reagent. The reaction was incubated for $1 \mathrm{~h}$ at room temperature. Hydroxylamine $(5 \%, 8 \mu \mathrm{L})$ was added to each sample, and the reactions were quenched over a 15 min period. Samples were combined in equal amounts in fresh microcentrifuge tubes, and the mixed samples were divided into eight fractions using the Pierce ${ }^{\mathrm{TM}}$ High $\mathrm{pH}$ ReversedPhase Peptide Fractionation Kit.

\section{LC-MS/MS analysis and database searching}

LC-MS/MS analysis was carried out using a Dionex Ultimate 3000 Nano LC system coupled with a Q-Exactive mass spectrometer (Thermo Fisher Scientific, USA) equipped with an electrospray ionization nanospray source. Mobile phases A and B were $0.1 \%$ FA in water and ACN, respectively. The total flow rate was $600 \mathrm{~nL} /$ min and a 120-min gradient was set as follows: from 4 to $10 \%$ B for $5 \mathrm{~min}$, from 10 to $22 \%$ B for $80 \mathrm{~min}$, from 22 to $40 \%$ B for $25 \mathrm{~min}$, from 40 to $95 \%$ B for $5 \mathrm{~min}$, and held at $95 \% \mathrm{~B}$ for $5 \mathrm{~min}$. The spray voltage was set at $2.0 \mathrm{kV}$. All MS and MS/MS spectra were acquired in data-dependent acquisition mode and the full mass scan was acquired from $m / z 300$ to 1400 with resolution of 70,000.

The raw MS files (The mass spectrometry proteomics data have been deposited to the ProteomeXchange Consortium via the PRIDE partner repository 
with the dataset identifier PXD022220 and https://doi. org/10.6019/pxd022220) were analyzed and searched against the UniProt $M$. pneumoniae database using Proteome Discover 2.1 (Thermo Fisher Scientific). Trypsin was selected as the enzyme and up to two missed cleavages were allowed. Cysteine residue alkylation was set as the static modification, and methionine oxidation was set as the variable modification. The mass tolerance of the precursor was $15 \mathrm{ppm}$ and the peptide false discovery rate was controlled at $\leq 1 \%$.

\section{Bioinformatics analysis}

We investigated the Gene Ontology (GO) and Kyoto Encyclopedia of Genes and Genomes (KEGG) pathways of the DEPs using the online OmicsBean resource (http:// www.omicsbean.cn/).

\section{Parallel reaction monitoring analysis}

The protein expression levels obtained from the TMT analysis were confirmed by quantifying the expression levels of five selected proteins using Parallel Reaction Monitoring (PRM) analysis. Unique peptides from the target proteins were defined according to the TMT data. The proteins $(50 \mu \mathrm{g})$ were prepared and digested following the TMT analysis protocol. The obtained peptide mixtures were analyzed by nano LC-PRM MS using easy nano-LC (Thermo Fisher Scientific) coupled to a Q Exactive $^{\mathrm{TM}}$ Hybrid Quadrupole-Orbitrap ${ }^{\mathrm{TM}}$ Mass Spectrometer (Thermo Fisher Scientific). The raw data were processed using Skyline 2.6, with the cut-off value set to 0.99 . The five product ions with the highest signal intensities were allowed to enter each peptide segment for analysis. Each peptide segment was manually integrated, and the results were exported for data analysis.

\section{Statistical analysis}

Statistical analysis was performed using SPSS Statistics software v22.0. Differences in the expression levels of six selected DEPs in the PRM analysis between the macrolide-resistant M. pneumoniae strain C267 and the macrolide-sensitive $M$. pneumoniae strain M129 were determined using a $t$-test, and $p<0.10$ was taken to indicate statistical significance.

\section{Results}

\section{Identification of DEPs}

TMT-labeled quantitative proteomes were determined for M. pneumoniae strains C267 and M129. Altogether, 7263 peptides corresponding to 531 proteins were detected. To ensure the reliability of the identification results, we performed peptide length and peptide matching error distribution analyses. Most of the identified peptides were 8-30 amino acids long, and therefore suitable for mass spectrometry (Additional file 1: Figure $\mathrm{S} 1 \mathrm{~A})$. The mass error rate for the peptides was $\pm 20 \mathrm{ppm}$, which confirmed that the identification was accurate (Additional file 1: Figure S1B).

A strict comparison at fold-change $\geq 1.2$ (for upregulation) or $\leq 1 / 1.2$ (for downregulation) and a $p$-value cutoff of $\leq 0.05$ was applied to identify the DEPs. When comparing macrolide-resistant strain (C267) with macrolidesensitive strain (M129), 165 DEPs were observed, with 80 upregulated and 85 downregulated (Additional file 2: Table S1). As shown in Fig. 1a and b, a volcano plot and heatmap were employed to analyze the DEPs.

\section{Functional categorization of DEPs}

A broad overview of the main differences between the two strains was obtained in the GO and KEGG analyses. We obtained the top 10 significant GO terms for DEPs in the following categories: biological process (BP), cellular component (CC), and molecular function (MF) (Fig. 2). Translation, cellular protein metabolic process, peptide biosynthetic process, peptide metabolic process, amide biosynthetic process, cellular amide metabolic process, cellular macromolecule biosynthetic process, and macromolecule biosynthetic process were the most significantly enriched in the BP category. In the CC category, cytoplasmic part, ribosome, intracellular ribonucleoprotein complex, ribonucleoprotein complex, and macromolecular complex were found to be significantly enriched. Notably, proton-transporting ATP synthase complex, coupling factor $\mathrm{F}(\mathrm{o})$, proton-transporting two-sector ATPase complex, and proton-transporting domain, were among the top 10 significantly enriched CC terms. In the MF category, the following DEPs were highly enriched: structural molecule activity, structural constituent of ribosome, monovalent inorganic cation transmembrane transporter activity, and inorganic cation transmembrane transporter activity terms.

Mycoplasmas have no cell wall and a small genome; therefore, they lack many enzyme activities found in most bacteria. The absent genes and enzyme function can be supplemented by other genes and their expression products. For these reasons, it is problematic to predict metabolic pathways only by protein annotation, proteome analysis and structural analysis (Pollack 2002). Thirtytwo KEGG pathways were annotated for the 165 DEPs (Additional file 3: Table S2). However, it was reported that many metabolic pathways, particularly biosynthetic pathways, are absent in Mycoplasmas, such as those participated in de novo purine biosynthesis and the biosynthesis of amino acids (Pollack et al. 1997; Barile et al. 1966; Himmelreich et al. 1996). They also lack TCA cycle pathway, because they are strict with the nutritional environment, which can be provided by their hosts (Franciele 
a

volcano_plot

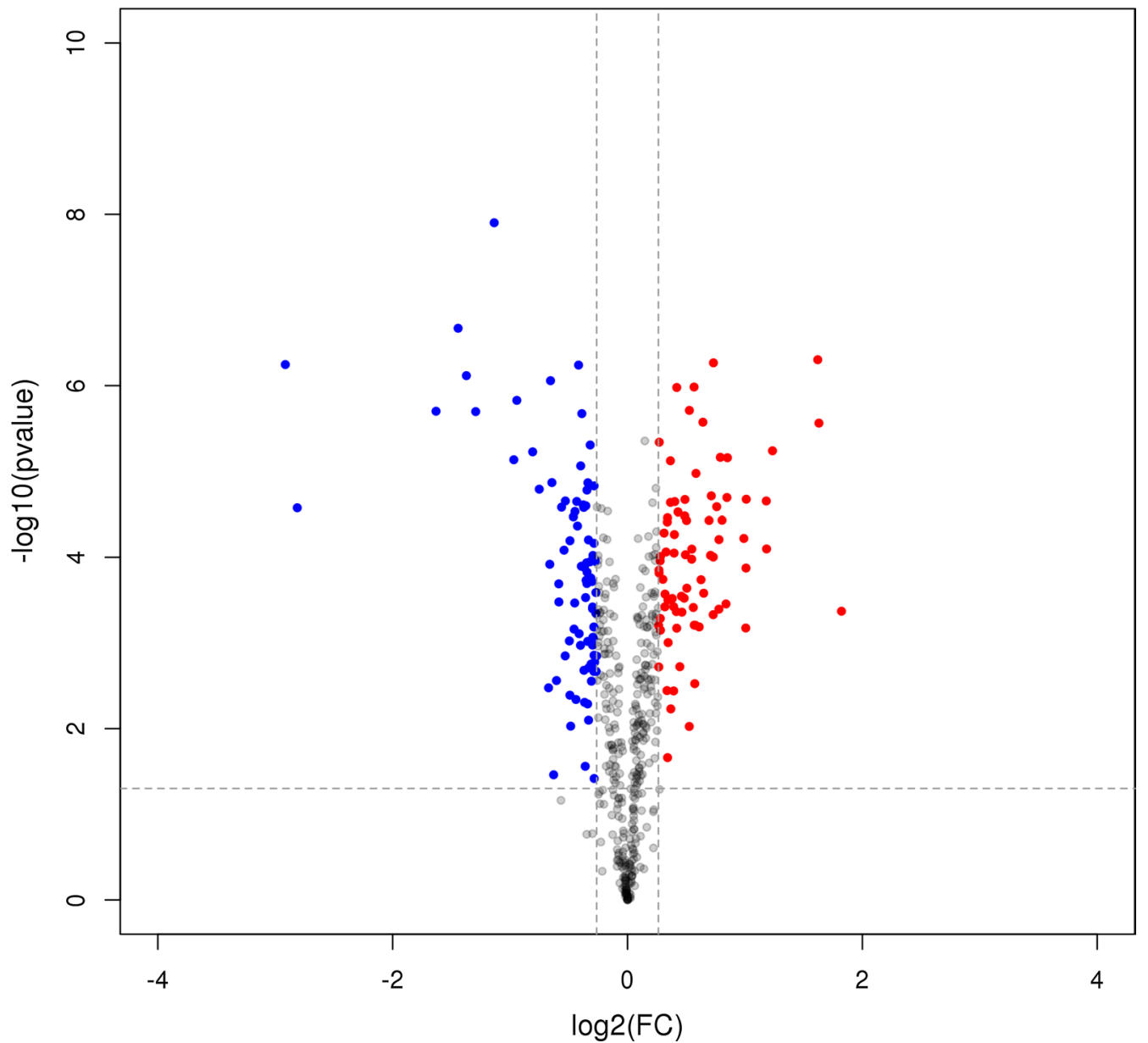

Fig. 1 Identification of differentially expressed proteins (DEPs). a Volcano plots of the DEPs. The horizontal coordinate indicates the log2(FC) values and the vertical coordinate indicates the $-\log 10$ values. $\mathbf{b}$ Heatmap of DEPs. The expression values shown in different colors denote different protein expression levels

et al. 2013). Table 1 showed the five pathways with the most DEPs. Ribosome pathway was the most significant and abundant pathway. Twenty-eight DEPs were involved with ribosome pathway, including rpsZ, rplW, rpsB, rpsC, rplL, rpsH, rplO, rplU, rplJ, rplK, rplF, rpsD, rplD, rplA, rpmC, rpmB, rplN, rplP, rplV, rplM, rpsT, rplC, rplE, rplX, rpsG, rpmE, rplQ and rpmA. EcfA2, ecfA1, MPN_611, pstA, potC, potA and MPN_058 participated in $\mathrm{ABC}$ transporters. A protein interaction network was constructed for the DEPs (Fig. 3). These interactions provide important information about the function and behavior of the DEPs, and are useful to comprehend the resistance mechanisms of M.pneumniae.

\section{Validation of DEPs}

Because antibodies suitable for use in M. pneumoniae are rare, targeted, quantitative MS approaches such as PRM and multireaction monitoring are essential for DEP confirmation. To validate the results obtained from TMTbased proteomics, we examined the expression levels of several candidate proteins by PRM. Because this technique requires the signature peptide of the target protein to be unique, we selected six proteins with unique signature peptide sequences for PRM analysis. The foldchanges for these proteins differed significantly between the macrolide-resistant C267 strain and the macrolidesensitive M129 strain at $p<0.05$, a result in agreement with the findings from the TMT analysis (Table 2). 


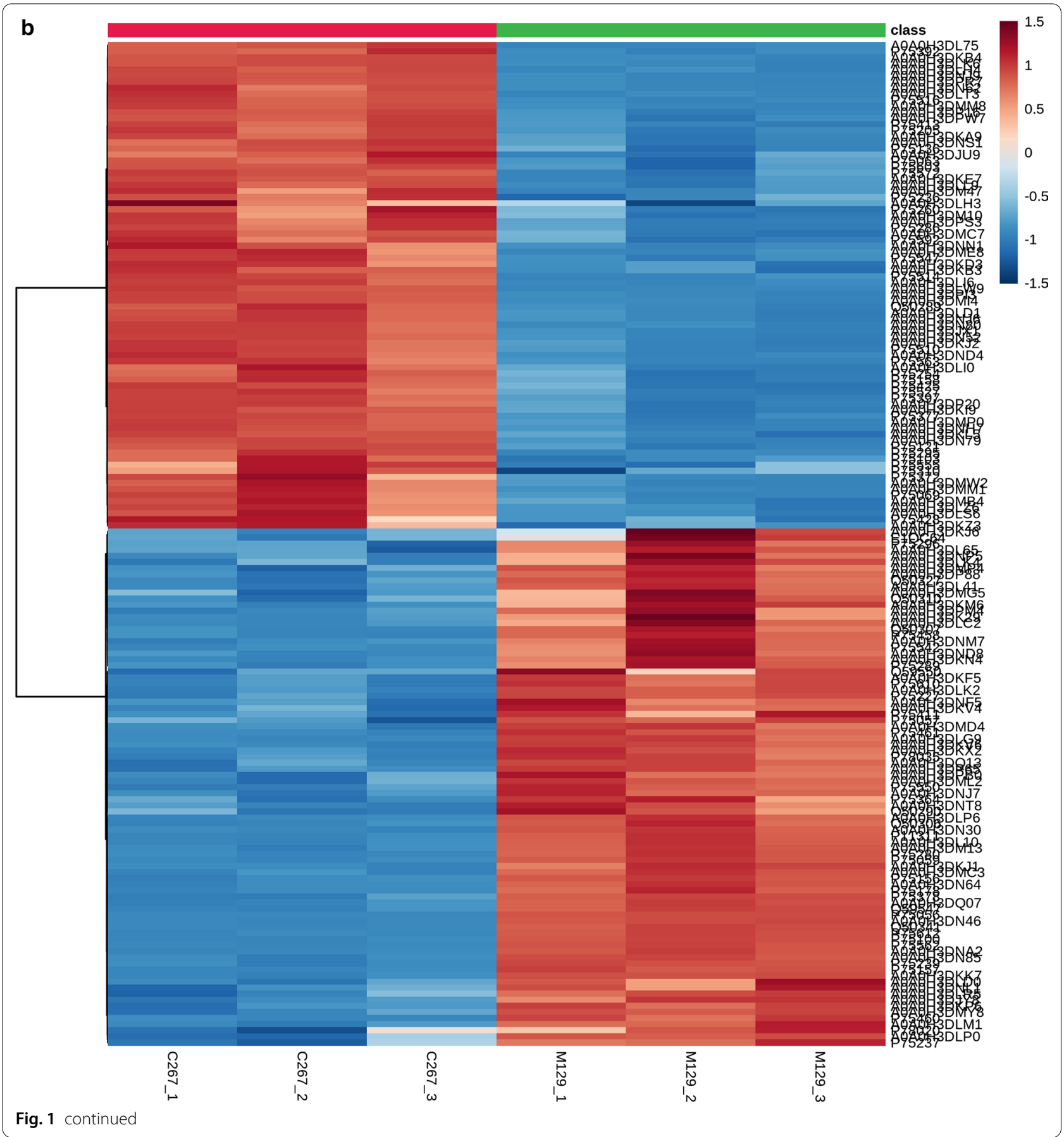

\section{Discussion}

Mycoplasma pneumoniae is one of the main pathogens to cause community-acquired respiratory tract infections and, because these infections can lead to bronchitis and atypical pneumonia as well as a variety of extrapulmonary complications, this pathogen can seriously endanger the health of children and adolescents (Uldum et al. 2012; Principi and Esposito 2013; Waites and Talkington 2004).
Because it lacks a cell wall, $M$. pneumoniae is resistant to $\beta$-lactams and other antibiotics that act on bacterial cell walls, but it is (in principle) sensitive to macrolides, tetracyclines, and quinolones, because these agents inhibit or affect the synthesis of bacterial proteins and nucleic acids. However, the increasing prevalence of macrolideresistant M. pneumoniae is a significant problem because clinical treatments depend on macrolide antibiotics 


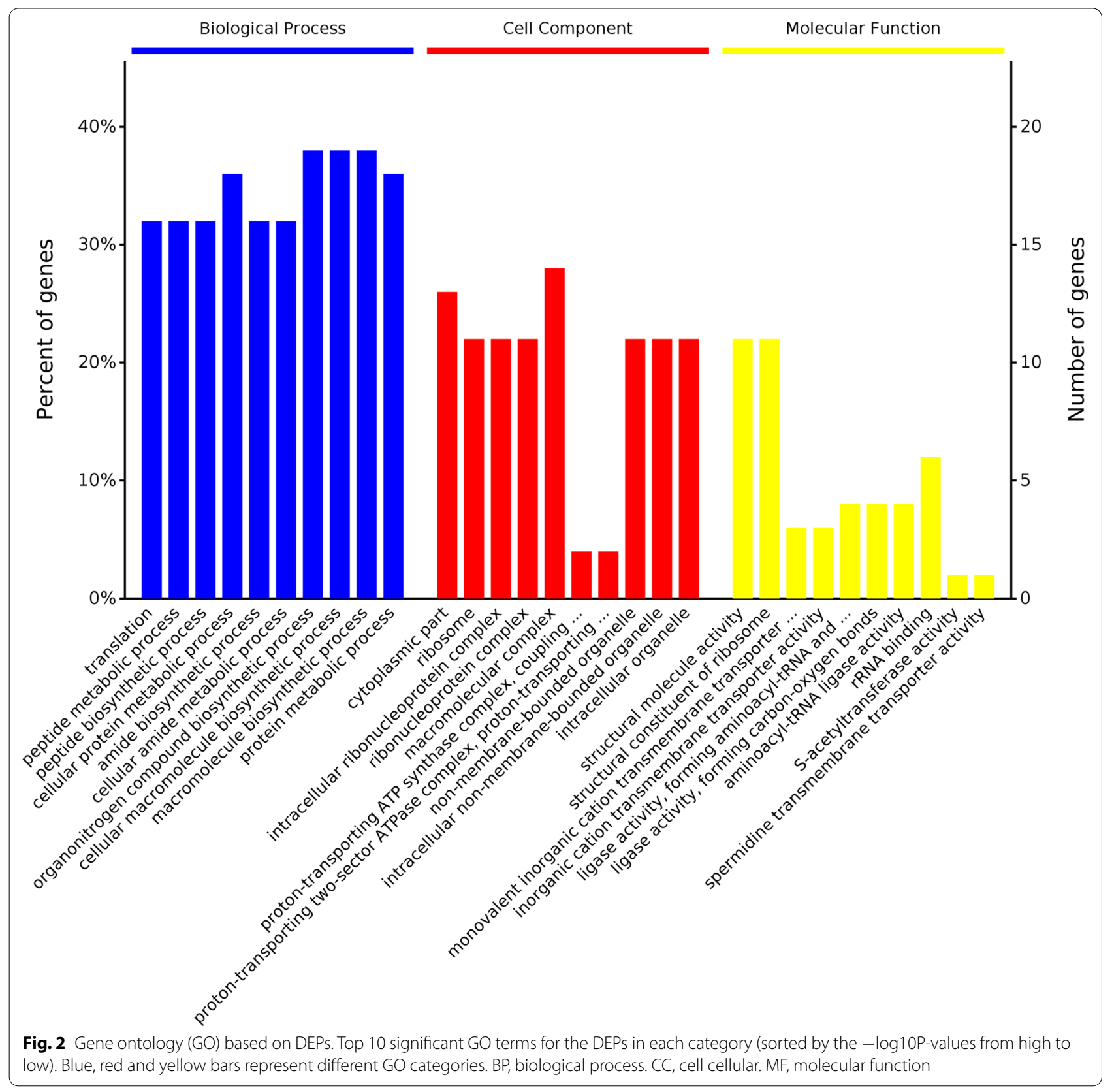

Table 1 KEGG annotation of DEPs between macrolide-resistant strain (C267) and macrolide-sensitive strain (M129)

\begin{tabular}{|c|c|c|c|}
\hline & Pathway Name & Count & Genes \\
\hline 1 & Ribosome & 28 & $\begin{array}{l}\text { rpsZ, rplW, rpsB, rpsC, rplL, rpsH, rplO, rplU, rplJ, rplK, rplF, rpsD, rplD, rplA, rpmC, rpmB, rpIN, rplP, rplV, rpIM, } \\
\text { rpsT, rplC, rplE, rplX, rpsG, rpmE, rpIQ, rpmA }\end{array}$ \\
\hline 2 & Metabolic pathways & 22 & $\begin{array}{l}\text { deoD, dnaX, plsY, glpK, tmk, pdhC, csd, ulaE, atpF, nrdE, atpG, MPN_450, rpoE, atpA, pyrH, nrdF, thyA, thil, } \\
\text { ulaF, tpiA, atpE, nadK }\end{array}$ \\
\hline 3 & Pyrimidine metabolism & 9 & deoD, dnaX, tmk, nrdE, MPN_450, rpoE, pyrH, nrdF, thyA \\
\hline 4 & Aminoacyl-tRNA biosynthesis & 8 & hisS, valS, trpS, glyQS, leuS, pheT, metG, alaS \\
\hline 5 & ABC transporters & 7 & ecfA2, ecfA1, MPN_611, pstA, potC, potA, MPN_058 \\
\hline
\end{tabular}




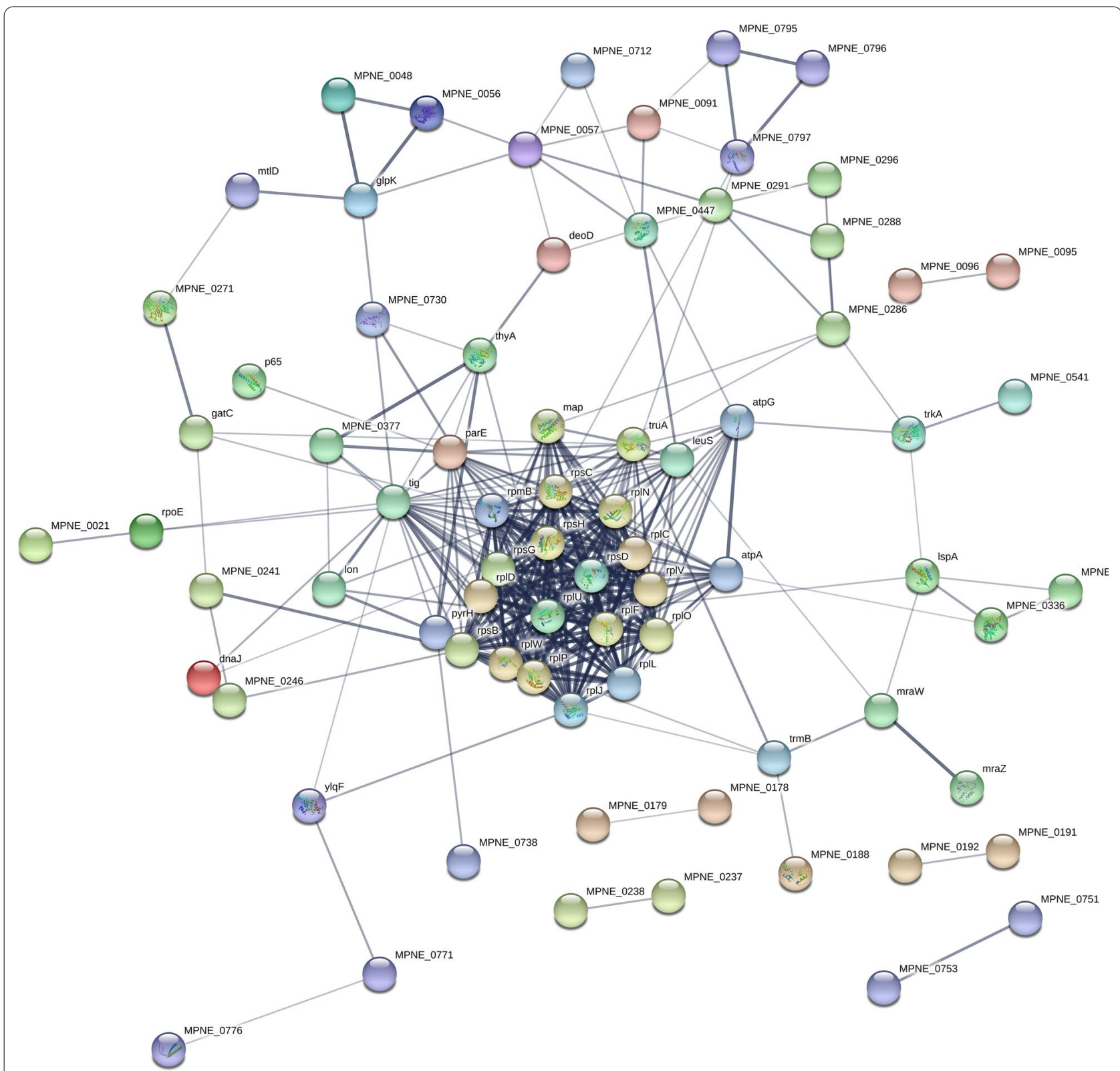

Fig. 3 STRING protein network analysis on the proteins identified as being significantly differentiated. Proteins were considered significant at a $p$-value of $<0.05$. The thicker the connecting lines in between the proteins the stronger the protein-protein associations

Table 2 Confirmation of the DEPs detected in the TMT analysis using PRM analysis

\begin{tabular}{|c|c|c|c|c|c|}
\hline Accession no. & Gene symbol & $\begin{array}{l}\text { Fold-change (C267/ } \\
\text { M129) in PRM }\end{array}$ & P-value in PRM & $\begin{array}{l}\text { Fold-change (C267/ } \\
\text { M129) in TMT }\end{array}$ & P-value in TMT \\
\hline P75295 & MPN_491 & 12.51738715 & $1.14223 \mathrm{E}-05$ & 2.268532831 & 2.22037E-05 \\
\hline P75121 & MPN_670 & 1.766381125 & 0.028324373 & 1.266310644 & $3.47397 \mathrm{E}-05$ \\
\hline P75603 & MPN_090 & 1.654676801 & 0.021731425 & 1.231982961 & 0.000180854 \\
\hline P75392 & pdhC & 1.549656015 & 0.009815993 & 1.240981292 & $5.22141 \mathrm{E}-05$ \\
\hline P75527 & def & 1.514331423 & 0.036288214 & 1.302137569 & 0.000302493 \\
\hline P75236 & MPN_542 & 1.37969945 & 0.098842902 & 1.493066835 & 0.000621553 \\
\hline
\end{tabular}


(Suzuki et al. 2013; Peuchant et al. 2009; Wolff et al. 2008; Dumke et al. 2010; Bajantri et al. 2018). Moreover, some studies have indicated that patients infected with macrolide-resistant strains have greater clinical manifestations and longer disease durations than those infected with wild-type (sensitive) strains (Zhou et al. 2014; Liu et al. 2010). Therefore, research into the drug resistance mechanism(s) of $M$. pneumoniae and implementing rational clinical drug use is now an urgent priority.

Macrolides bind to ribosomal subunits from bacteria and inhibit protein synthesis by blocking peptide transfer and mRNA displacement (Roberts 2004; Giedraitienè et al. 2011). Previous studies on M. pneumoniae resistance focused on point mutations in the $23 \mathrm{~S}$ ribosomal gene and ribosomal proteins L4 and L22 (Principi and Esposito 2013; Matsuoka et al. 2004; Suzuki et al. 2006). Our previous study confirmed that macrolide-resistant strain C267 harbors an A to G mutation at nucleotide position 2063 within domain $\mathrm{V}$ of the $23 \mathrm{~S}$ rRNA gene ( $\mathrm{Li}$ et al. 2017). In the present study, the GO analysis of DEPs showed that translation, peptide biosynthetic processes, ribosome, intracellular ribonucleoprotein complex, ribonucleoprotein complex, and the structural constituents of ribosomes were significantly enriched terms in the macrolide-resistant strain when compared with the sensitive strain (Fig. 3). The KEGG analysis also revealed that 28 DEPs were involved in ribosomal pathways (Table 1). Notably, all the ribosomal proteins were downregulated in the resistant strain unlike those in the sensitive strain (Fig. 4). Saito et al. (1969) found that erythromycin-ribosome complex formation decreased in erythromycinresistant Staphylococcus aureus strains (Saito et al. 1969). The reduced ability of ribosomes from resistant cells to bind erythromycin and other macrolide antibiotics has been used to demonstrate induced resistance, as has the increased resistance of these ribosomes to inhibition by macrolide antibiotics in cell-free protein synthesis (Shimizu et al. 1970; Weisblum et al. 1971; Allen 1977). Therefore, ribosomal proteins play an important role in drug resistance in M. pneumoniae. However, the exact mechanism(s) underpinning the involvement of ribosomal proteins in drug resistance need(s) to be investigated further.

Transporters are another important type of protein involved in drug resistance. Transporters pump a drug out of the cell or the cellular membrane, thereby keeping the intercellular concentrations low (Roberts 2004). An active efflux system, possibly an ABC-type efflux pump, was suggested to be involved in resistance to ciprofloxacin in wall-less $M$. hominis (Raherison et al. 2002). The existence of an active efflux process in $M$. hominis was also implicated in resistance to erythromycin because an $\mathrm{ABC}$ transporter inhibitor was able to increase erythromycin uptake levels by more than twofold (Pereyre et al. 2002). Our previous study showed that a macrolide efflux pump, possibly an ABC-type efflux pump, may contribute to macrolide resistance in M. pneumoniae C267 ( $\mathrm{Li}$ et al. 2017). The proteomics results from the present study support this assumption. KEGG analysis of the DEPs between the macrolide-resistant strain $\mathrm{C} 267$ and macrolide-sensitive strain M129 indicates that the following seven DEPs are associated with $A B C$ transporters: ECFA2, ECFA1, MPN_611, PSTA, POTC, POTA and MPN_058. In addition, spermidine transmembrane transporter activity, monovalent inorganic cation transmembrane transporter activity, and inorganic cation transmembrane transporter activity were among the 10 most significantly enriched MF terms, suggesting that transporters are significant players in macrolide resistance in $M$. pneumoniae.

Cell membranes consist of a lipid bilayer in which proteins that have important cellular functions, such as receptors, transporters, and enzymes, are embedded (Spector and Yorek 1985). The cell membranes play an important role by acting as a permeability barrier to the entry of diverse chemical agents (Nikaido 2003). Alteration of the cell membrane's lipid composition can be related to drug resistance. Changed membrane phospholipid and sterol compositions were observed in both clinical and in vitro-adapted azole-resistant Candida albicans isolates (Mukhopadhyay et al. 2002; Hitchcock et al. 1986; Kohli et al. 2002; Löffler et al. 2000). It was reported that benzyldimethyltetradecylammonium chloride-adapted Pseudomonas aeruginosa cells showed variations in membrane fatty acid composition (Nikaido 2003). In our analysis, two lipid-related pathways, glycerolipid metabolism and glycerophospholipid metabolism, were altered in resistant $M$. pneumoniae C267 compared with sensitive M. pneumoniae M129 (Additional file 2: Table S1). Such changes are possibly one of the causes of $M$. pneumoniae drug resistance and, as such, they warrant further investigation.

Drug resistance in $M$. pneumoniae is an increasingly serious problem, and further research into the mechanisms underlying it in this bacterium is urgently needed. Our study provides a global analysis of protein expression changes between the macrolide-resistant C267 strain and the macrolide-sensitive M129 strain of $M$. pneumoniae. We identified several important pathways and candidate proteins that are potential targets for further studies on macrolide resistance in $M$. pneumoniae. However, one limitation of this study is that the sample size was too low. Therefore, our future goal is to test more strains to confirm the relationship 


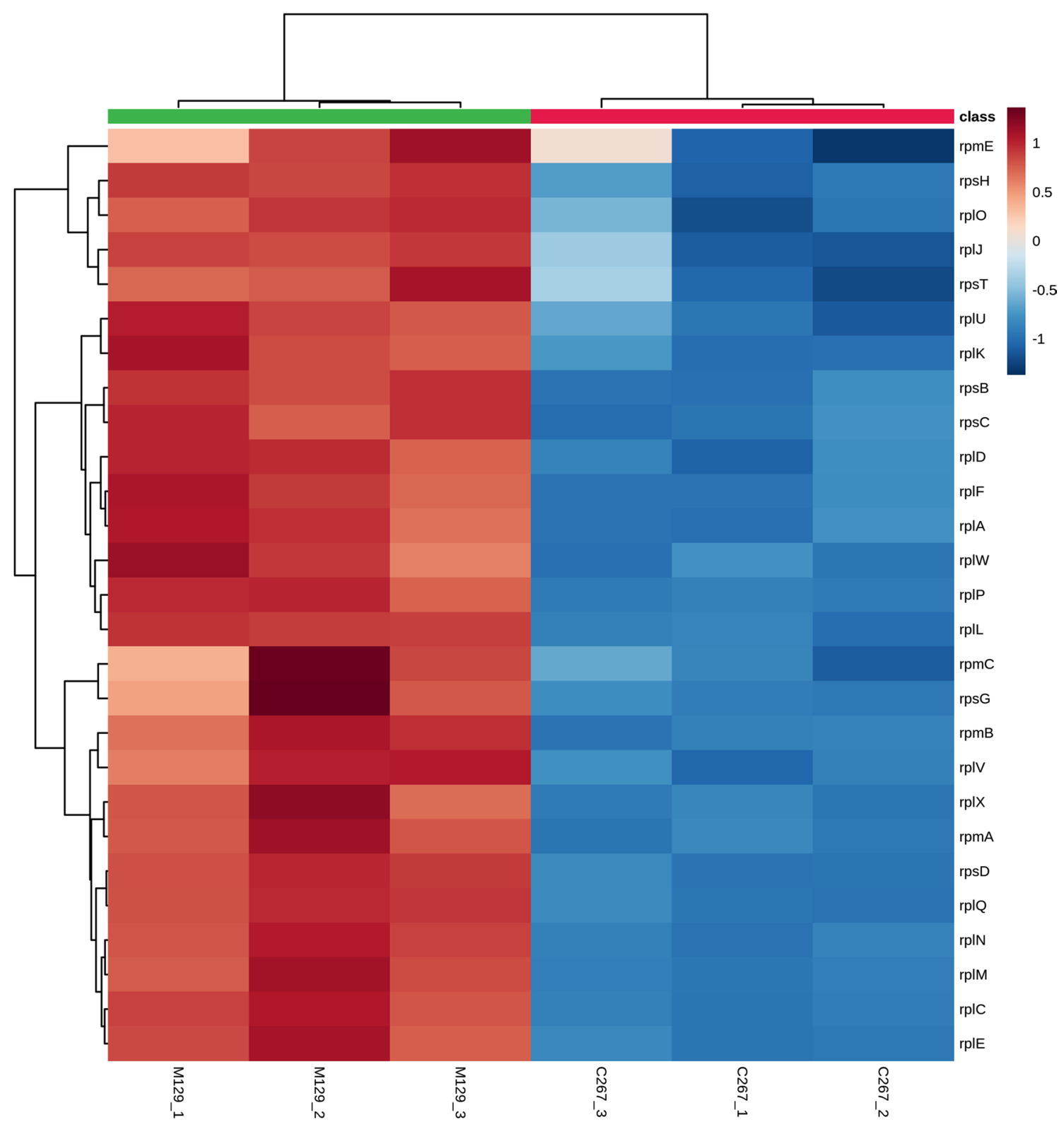

Fig. 4 Heatmap of ribosome proteins. The expression values shown in different colors denote the different protein expression levels

between protein expression and drug resistance in $M$. pneumoniae.

\section{Supplementary Information}

The online version contains supplementary material available athttps://doi. org/10.1186/s13568-021-01187-8.

Additional file 1: Figure S1. Quality control for the mass spectrometry identification process. The peptide length distribution (A) and the mass error distribution (B) are shown.

Additional file 2: Table S1. 165 differentially expressed proteins were identified in this study.
Additional file 3: Table S2. All KEGG annotation result of DEPs between macrolide-resistantstrain (C267) and macrolide-sensitivestrain (M129).

\section{Authors' contributions}

$\mathrm{SL}$ and GX conceived and designed the research. $\mathrm{HZ}$ and $\mathrm{YF}$ conducted the experiments. CY and JC contributed new reagents or analytical tools. SL and JY analyzed the data. SL and XX wrote the manuscript. All authors read and approved the final manuscript.

\section{Funding}

This work was supported by the National Natural Science Foundation [32070188 and 81601778], Research Foundation of Capital Institute of Pediatrics [FX-2020-02] and CAMS innovation Fund for Medical Sciences (CIFMS) 
2016-12M-1-008. The funders had no role in the study design, data collection and interpretation or the decision to submit the work for publication.

\section{Availability of data and materials}

The datasets used and/or analyzed during the current study are available from the corresponding author on reasonable request.

\section{Code availability}

Not applicable

\section{Ethics approval and consent to participate}

The present study was performed in compliance with the Helsinki Declaration (Ethical Principles for Medical Research Involving Human Subjects) and was approved by the research board of the Ethics Committee of the Capital Institute of Pediatrics, Beijing, China. The patient data were anonymously reported. Based on the guidelines of the Ethics Committee of the Capital Institute of Pediatrics, informed consent was not sought from the patients.

\section{Consent for publication}

Not applicable.

\section{Competing interests}

The authors declare that there are no competing interests associated with this manuscript.

Received: 5 November 2020 Accepted: 4 February 2021

Published online: 12 February 2021

\section{References}

Allen NE (1977) Macrolide resistance in Staphylococcus aureus: induction of macrolide-resistant protein synthesis. Antimicrob Agents Chemother 11:661-668. https://doi.org/10.1128/aac.11.4.661

Bajantri B, Venkatram S, Diaz-Fuentes G (2018) Mycoplasma pneumoniae: a potentially severe infection. J Clin Med Res 10:535-544. https://doi. org/10.14740/jocmr3421w

Barile MF, Schimke RT, Riggs DB (1966) Presence of arginine dihydrolase pathway in Mycoplasma. J Bacteriol 91:189-192. https://doi.org/10.1128/ JB.91.1.189-192.1966

Cao B, Qu JX, Yin YD, Eldere JV (2017) Overview of antimicrobial options for Mycoplasma pneumoniae pneumonia: focus on macrolide resistance. Clin Respir J 11:419-429. https://doi.org/10.1111/crj.12379

Dumke R, von Baum H, Lück PC, Jacobs E (2010) Occurrence of macrolideresistant Mycoplasma pneumoniae strains in Germany. Clin Microbiol Infect 16:613-616. https://doi.org/10.1111/j.1469-0691.2009.02968.x

Franciele MS, Thompson CE, Virginio VG, Gonchoroski T, Reolon L, Almeida LG, da Fonsêca MM, de Souza R, Prosdocimi F, Schrank IS, Ferreira HB (2013) New insights on the biology of swine respiratory tract mycoplasmas from a comparative genome analysis. BMC Genomics 14:175. https://doi. org/10.1186/1471-2164-14-175

Giedraitienè A, Vitkauskienè A, Naginienè R, Pavilonis A (2011) Antibiotic resistance mechanisms of clinically important bacteria. Medicina (Kaunas) 47(3):137-146

Himmelreich R, Hilbert H, Plagens H, Pirkl E, Li BC, Herrmann R (1996) Complete sequence analysis of the genome of the bacterium Mycoplasma pneumoniae. Nucleic Acids Res 24:4420-4449. https://doi.org/10.1093/ nar/24.22.4420

Hitchcock CA, Barrett-Bee KJ, Russell NJ (1986) The lipid composition of azolesensitive and azole-resistant strains of Candida albicans. J Gen Microbiol 132:2421-2431. https://doi.org/10.1099/00221287-132-9-2421

Kohli A, Smriti Mukhopadhyay K, Rattan A, Prasad R (2002) In vitro low-level resistance to azoles in Candida albicans is associated with changes in membrane lipid fluidity and asymmetry. Antimicrob Agents Chemother 46:1046-1052. https://doi.org/10.1128/aac.46.4.1046-1052.2002

Lee H, Yun KW, Lee HJ, Choi EH (2018) Antimicrobial therapy of macrolideresistant Mycoplasma pneumoniae pneumonia in children. Expert Rev Anti Infect Ther 16:23-34. https://doi.org/10.1080/14787210.2018.14145 99

Li S, Sun H, Liu F, Zhao H, Zhu B, Lv N (2016) Complete genome sequence of the macrolide-resistant Mycoplasma pneumoniae strain C267 in China. Genome Announc 4:e00236. https://doi.org/10.1128/genomeA.00236-16
Li SL, Sun HM, Zhu BL, Liu F, Zhao HQ (2017) Whole genome analysis reveals new insights into macrolide resistance in Mycoplasma pneumoniae. Biomed Environ Sci 30:343-350. https://doi.org/10.3967/bes2017.045

Liu Y, Ye X, Zhang H, Xu X, Li W, Zhu D, Wang M (2010) Characterization of macrolide resistance in Mycoplasma pneumoniae isolated from children in Shanghai, China. Diagn Microbiol Infect Dis 67:355-358. https://doi. org/10.1016/j.diagmicrobio.2010.03.004

Löffler J, Einsele H, Hebart H, Schumacher U, Hrastnik C, Daum G (2000) Phospholipid and sterol analysis of plasma membranes of azole-resistant Candida albicans strains. FEMS Microbiol Lett 185:59-63. https://doi. org/10.1111/j.1574-6968.2000.tb09040.x

Matsuoka M, Narita M, Okazaki N, Ohya H, Yamazaki T, Ouchi K, Suzuki I, Andoh T, Kenri T, Sasaki Y, Horino A, Shintani M, Arakawa Y, Sasaki T (2004) Characterization and molecular analysis of macrolide-resistant Mycoplasma pneumoniae clinical isolates obtained in Japan. Antimicrob Agents Chemother 48:4624-4630. https://doi.org/10.1128/ AAC.48.12.4624-4630.2004

Mukhopadhyay K, Kohli A, Prasad R (2002) Drug susceptibilities of yeast cells are affected by membrane lipid composition. Antimicrob Agents Chemother 46:3695-3705. https://doi.org/10.1128/aac.46.12

Nikaido H (2003) Molecular basis of bacterial outer membrane permeability revisited. Microbiol Mol Biol Rev 67(4):593-656. https://doi.org/10.1128/ mmbr.67.4.593-656.2003

Pereyre S, Gonzalez P, De Barbeyrac B, Darnige A, Renaudin H, Charron A, Raherison S, Bébéar C, Bébéar CM (2002) Mutations in 23S rRNA account for intrinsic resistance to macrolides in Mycoplasma hominis and Mycoplasma fermentans and for acquired resistance to macrolides in M. hominis. Antimicrob Agents Chemother 46:3142-3150. https://doi. org/10.1128/aac.46.10.3142-3150.2002

Pereyre S, Goret J, Bébéar, C (2016) Mycoplasma pneumoniae: current knowledge on macrolide resistance and treatment. Front Microbiol 7:974. https ://doi.org/10.3389/fmicb.2016.00974

Peuchant O, Ménard A, Renaudin H, Morozumi M, Ubukata K, Bébéar CM, Pereyre S (2009) Increased macrolide resistance of Mycoplasma pneumoniae in France directly detected in clinical specimens by real-time PCR and melting curve analysis. J Antimicrob Chemother 64:52-58. https:// doi.org/10.1093/jac/dkp160

Pollack JD (2002) The necessity of combining genomic and enzymatic data to infer metabolic function and pathways in the smallest bacteria: amino acid, purine and pyrimidine metabolism in Mollicutes. Front Biosci 7:d1762-d1781

Pollack JD, Williams MV, Mcelhaney RN (1997) The comparative metabolism of the mollicutes (Mycoplasmas): the utility for taxonomic classification and the relationship of putative gene annotation and phylogeny to enzymatic function in the smallest free-living cells. Crit Rev Microbiol 23:269-354. https://doi.org/10.3109/10408419709115140

Principi N, Esposito S (2013) Macrolide-resistant Mycoplasma pneumoniae: its role in respiratory infection. J Antimicrob Chemother 68:506-511. https:// doi.org/10.1093/jac/dks457

Raherison S, Gonzalez P, Renaudin H, Charron A, Bébéar C, Bébéar CM (2002) Evidence of active efflux in resistance to ciprofloxacin and to ethidium bromide by Mycoplasma hominis. Antimicrob Agents Chemother 46:672-679. https://doi.org/10.1128/aac.46.3.672-679.2002

Roberts MC (2004) Resistance to macrolide, lincosamide, streptogramin, ketolide, and oxazolidinone antibiotics. Mol Biotechnol 28:47-62. https:// doi.org/10.1385/MB:28:1:47

Saito T, Hashimoto H, Mitsuhashi S (1969) Drug resistance of staphylococci. Foation of erythromycin-ribosome complex. Decrease in the formation of erythromycin-ribosome complex in erythromycin resistant strains of Staphylococcus aureus. Jpn J Microbiol 13:119-121. https://doi. org/10.1111/j.1348-0421.1969.tb00441.x

Saraya T (2016) The History of Mycoplasma pneumoniae Pneumonia. Front Microbiol 7:364. https://doi.org/10.3389/fmicb.2016.00364

Shimizu M, Saito T, Mitsuhashi S (1970) Macrolide resistance in Staphylococcus aureus. Correlation between spiramycin-binding to ribosomes and inhibition of polypeptide synthesis in cell-free system. Jpn J Microbiol 14:215-219. https://doi.org/10.1111/j.1348-0421.1970.tb00512.x

Spector AA, Yorek MA (1985) Membrane lipid composition and cellular function. J Lipid Res 26:1015-1035

Suzuki S, Yamazaki T, Narita M, Okazaki N, Suzuki I, Andoh T, Matsuoka M, Kenri T, Arakawa Y, Sasaki T (2006) Clinical evaluation of macrolide-resistant 
Mycoplasma pneumoniae. Antimicrob Agents Chemother 50:709-712. https://doi.org/10.1128/AAC.50.2.709-712.2006

Suzuki Y, Itagaki T, Seto J, Kaneko A, Abiko C, Mizuta K, Matsuzaki Y (2013) Community outbreak of macrolide-resistant Mycoplasma pneumoniae in Yamagata, Japan in 2009. Pediatr Infect Dis J 32(3):237-240. https://doi. org/10.1097/INF.0b013e31827aa7bd

Tanaka T, Oishi T, Miyata I, Wakabayashi S, Kono M, Ono S, Kato A, Fukuda Y, Saito A, Kondo E, Teranishi H, Tanaka Y, Wakabayashi T, Akaike H, Ogita S, Ohno N, Nakano T, Terada K, Ouchi K (2017) Macrolide-resistant Mycoplasma pneumoniae infection, Japan, 2008-2015. Emerg Infect Dis 23:1703-1706. https://doi.org/10.3201/eid2310.170106

Uldum SA, Bangsborg JM, Gahrn-Hansen B, Ljung R, Mølvadgaard M, Føns Petersen R, Wiid Svarrer C (2012) Epidemic of Mycoplasma pneumoniae infection in Denmark, 2010 and 2011. Euro Surveill 17:20073. https://doi. org/10.2807/ese.17.05.20073-en

Waites KB, Talkington DF (2004) Mycoplasma pneumoniae and its role as a human pathogen. Clin Microbiol Rev 17:697-728. https://doi. org/10.1128/cmr.17.4.697-728.2004 table of contents

Waites KB, Xiao L, Liu Y, Balish MF, Atkinson TP (2017) Mycoplasma pneumoniae from the respiratory tract and beyond. Clin Microbiol Rev 30:747-809. https://doi.org/10.1128/CMR.00114-16
Weisblum B, Siddhikol C, Lai CJ, Demohn V (1971) Erythromycin-inducible resistance in Staphylococcus aureus: requirements for induction. J Bacteriol 106:835-847. https://doi.org/10.1128/JB.106.3.835-847.1971

Wolff BJ, Thacker WL, Schwartz SB, Winchell JM (2008) Detection of macrolide resistance in Mycoplasma pneumoniae by real-time PCR and high-resolution melt analysis. Antimicrob Agents Chemother 52:3542-3549. https:// doi.org/10.1128/AAC.00582-08

Yang HJ, Song DJ, Shim JY (2017) Mechanism of resistance acquisition and treatment of macrolide-resistant Mycoplasma pneumoniae pneumonia in children. Korean J Pediatr 60:167-174. https://doi.org/10.3345/ kjp.2017.60.6.167

Zhou Y, Zhang Y, Sheng Y, Zhang L, Shen Z, Chen Z (2014) More complications occur in macrolide-resistant than in macrolide-sensitive Mycoplasma pneumoniae pneumonia. Antimicrob Agents Chemother 58:1034-1038 https://doi.org/10.1128/AAC.01806-133695-3705.2002

\section{Publisher's Note}

Springer Nature remains neutral with regard to jurisdictional claims in published maps and institutional affiliations.

\section{Submit your manuscript to a SpringerOpen ${ }^{\circ}$ journal and benefit from:}

- Convenient online submission

- Rigorous peer review

- Open access: articles freely available online

- High visibility within the field

- Retaining the copyright to your article

Submit your next manuscript at $\boldsymbol{\nabla}$ springeropen.com 